\title{
Sustitución del manganeso en las fases del clínker del cemento portland
}

\author{
Manganese substitutions into the portland cement \\ clinker phases
}

\author{
F. PUERTAS, $M^{a}$ TERESA BLANCO y T. VAZQUEZ \\ IETCC/CSIC \\ ESPAÑA
}

Fecha de recepción: 28-IV-1989

\section{RESUMEN}

Se ha estudiado, por difracción de rayos $X$ y microanálisis por espectroscopia de energias dispersivas, el efecto de la sustitución del manganeso en las estructuras cristalinas de las fases más importantes del clínker del cemento portland $\left(3 \mathrm{CaO} . \mathrm{SiO}_{2}, 2 \mathrm{CaO} . \mathrm{SiO}_{2}, 3 \mathrm{CaO} . \mathrm{Al}_{2} \mathrm{O}_{3}\right.$. $2 \mathrm{CaO} . \mathrm{Fe}_{2} \mathrm{O}_{3}$ y $4 \mathrm{CaO} \cdot \mathrm{Al}_{2} \mathrm{O}_{3}$. $\mathrm{Fe}_{2} \mathrm{O}_{3}$ ).

En condiciones oxidantes, el limite de solubilidad sólida en $3 \mathrm{CaO} . \mathrm{SiO}_{2}$ es del orden de $0,72 \pm 0,11 \%$ en peso; mientras que en $2 \mathrm{CaO}$. $\mathrm{SiO}_{2}$ es de $1,53 \pm 0,12 \%$ en peso.

La solución sólida del $\mathrm{Mn}$ en la estructura del $3 \mathrm{CaO} \cdot \mathrm{Al}_{2} \mathrm{O}_{3}$, en condiciones oxidantes, es próxima al 0,78 $\pm 0,12 \%$ en peso. En idéntica atmósfera, la proporción del Mn en las fases ferríticas ( $2 \mathrm{CaO} . \mathrm{Fe}_{2} \mathrm{O}_{3}$ y $4 \mathrm{CaO} . \mathrm{Al}_{2} \mathrm{O}_{3} . \mathrm{Fe}_{2} \mathrm{O}_{3}$ ) es de $6,80 \pm 0,87 \%$ en peso, y $6,7 \%$, respectivamente.

Se ha propuesto para cada una de las fases del clinker mencionadas, su correspondiente fórmula de solubilidad sólida. En dichas fórmulas se define el grado de sustitución y el estado de oxidación en el que el Mn se ha incorporado en las citadas estructuras cristalinas.

\begin{abstract}
The effect of manganese substitution into the crystai structures of the main Portland cement clinker phases $\left(3 \mathrm{CaO} . \mathrm{SiO}_{2}, 2 \mathrm{CaO} . \mathrm{SiO}_{2}, 3 \mathrm{CaO} . \mathrm{Al}_{2} \mathrm{O}_{3}, 2 \mathrm{CaO} . \mathrm{Fe}_{2} \mathrm{O}_{3}\right.$ and $4 \mathrm{CaO} \cdot \mathrm{Al}_{2} \mathrm{O}_{3} \cdot \mathrm{Fe}_{2} \mathrm{O}_{3}$ ) has been studied by $X$-ray and analytical electron microscopy.
\end{abstract}

In oxidizing conditions, the limit of solid solution in $3 \mathrm{CaO} . \mathrm{SiO}_{2}$ is about $0,72 \pm 0,11 \%$ (wt), while in $2 \mathrm{CaO}^{\mathrm{SiO}}$ is $1,53 \pm 0,12 \%(w t)$.

$\mathrm{Mn}$ solid solubility on $3 \mathrm{CaO} . \mathrm{Al}_{2} \mathrm{O}_{3}$ structure, in oxidizing conditions is close to $0,78 \pm 0,12 \%(w t)$. In identical atmosphere, the proportion of $\mathrm{Mn}$ in the ferrite phases $\left(2 \mathrm{CaO} . \mathrm{Fe}_{2} \mathrm{O}_{3}\right.$ and $4 \mathrm{CaO} \cdot \mathrm{Al}_{2} \mathrm{O}_{3} \cdot \mathrm{Fe}_{2} \mathrm{O}_{3}$ ) is $6,80 \pm 0,87 \%$ (wt) and $6,7 \%$ (wt), respectively.

To each mentioned clinker phases a solid solution formula has been proposed. In these formula, the manganese substitutions and also the different oxidation states which this element can be introduced in those crystalline structure, are defined.

\section{INTRODUCCION}

El contenido de oxidos de manganeso en el cemento portland raramente excede del $0,1-0,2 \%$ salvo en cementos que contienen escorias de alto horno, donde este valor puede alcanzar hasta un $5 \%$ (1). Por esta razón, es de gran interés conocer las modificaciones o alteraciones que dicho elemento produce en las estructuras cristalinas de los componentes más importantes del clínker del cemento portland $\left(3 \mathrm{CaO} . \mathrm{SiO}_{2}, 2 \mathrm{CaO} . \mathrm{SiO}_{2}, 3 \mathrm{CaO} . \mathrm{Al}_{2} \mathrm{O}_{3}\right.$, $2 \mathrm{CaO} . \mathrm{Fe}_{2} \mathrm{O}_{3}$ y $4 \mathrm{CaO} \cdot \mathrm{Al}_{2} \mathrm{O}_{3} \cdot \mathrm{Fe}_{2} \mathrm{O}_{3}$ ).

Existen algunos trabajos en torno a este tema; entre ellos cabria resaltar el realizado por Esculescu (2) quien indicó que el $\mathrm{MnO}_{2}$ se

\section{INTRODUCTION}

The content of manganese oxides in Portland cement rarely exceeds $0,1-0,2 \%$, although in cements containing blast-furnace slags it can be up to $5 \%$ (1). For this reason, it is of interest to know the modifications or alterations manganese may produce to the crystal structures of the main portland cement clinker phases $\left(3 \mathrm{CaO} . \mathrm{SiO}_{2}, 2 \mathrm{CaO} . \mathrm{SiO}_{2}, 3 \mathrm{CaO} . \mathrm{Al}_{2} \mathrm{O}_{3}\right.$, $2 \mathrm{CaO} . \mathrm{Fe}_{2} \mathrm{O}_{3}$ and $4 \mathrm{CaO} \cdot \mathrm{Al}_{2} \mathrm{O}_{3} . \mathrm{Fe}_{2} \mathrm{O}_{3}$ ).

There are some studies on this subject; it should be remarked the one carried out by Esculescu (2) who showed that $\mathrm{MnO}_{2}$ forms 
solubiliza en el $\mathrm{C}_{3} \mathrm{~S}$ en proporciones no superiores al $1 \%$ en peso. Este autor propuso la siguiente fórmula de solubilidad sólida: solid solutions based on $\mathrm{C}_{3} \mathrm{~S}$ up to $1 \%$. He proposed the following constitutional formulae for the solid solution:

$$
\left(\mathrm{Ca}_{3-x} \mathrm{Mn}_{\mathrm{x}}\right)\left(\mathrm{Si}_{1-y} \mathrm{Mn}_{\mathrm{y}}\right) \mathrm{O}_{z-2} \text {, }
$$

donde

$$
z=x+2 y-u(x+y)
$$

$x$ e y son las sustituciones por calcio y silicio respectivamente, y u es la valencia del catión metálico.

Recientemente, Knöfel (3) demostró que el límite en la sustitución del $\mathrm{MnO}_{2}$ en el silicato tricálcico a $1.550^{\circ} \mathrm{C}$ es próximo al $2,2 \%$ en peso. Además propuso un posible mecanismo de sustitución en donde $0,1 \%$ de $\mathrm{MnO}_{2}$ verifica una sustitución simple de $\mathrm{Si}^{4}$ : por $\mathrm{Mn}^{4+}$, por encima de este valor y hasta el 2,2 \%, se produce una sustitución doble de $\mathrm{Si}^{4+}$ por $\mathrm{Mn}^{4+}$ y $\mathrm{Ca}^{2+}$ por $\mathrm{Mn}^{2+}$. Las fórmulas de solubilidad propuestas por Knöfel fueron: $x$ and $y$ are the substitutions for calcium and silicon respectively and $u$ is the valence of the metallic cation.

Recently, Kröfel (3) indicated that the limit of $\mathrm{MnO}_{2}$ substitution, in tricalcium silicate is about $2.2 \%$ at $1.550^{\circ} \mathrm{C}$. Moreover, he proposed a possible substitution mechanism in which $0,1 \%$ of $\mathrm{MnO}_{2}$ occurs by single substitution of $\mathrm{Mn}^{4+}$ by $\mathrm{Si}^{4+}$, from this value up to $2.2 \%$ a double substitution of $\mathrm{Mn}^{4+}$ by $\mathrm{Si}^{4+}$ and $\mathrm{Mn}^{2+}$ by $\mathrm{Ca}^{2}$ : was considered more probable. The solution formulae proposed by Knöfel were:

y

$$
\mathrm{Ca}_{3}\left(\mathrm{Si}_{1-x} \mathrm{Mn}_{\mathrm{x}}\right) \mathrm{O}_{5} \mathrm{O} \leq \mathrm{x} \leq 0,003
$$

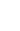

$$
\left(\mathrm{Ca}_{1.003-x} \mathrm{Mn}_{(\mathrm{x}-0,1015)}\right)_{3}\left(\mathrm{Si}_{0,997} \mathrm{Mn}_{0,003}\right) \mathrm{O}_{5} 0,003 \leq \mathrm{x} \leq 0,060
$$

Existe una controversia abierta en torno a que polimorfo del $\mathrm{C}_{3} \mathrm{~S}$ se estabiliza en presencia de manganeso. Algunos autores (4) indican que es la forma triclinica, aunque otros $(2,3)$ sostienen que es la forma monoclínica.

Esculescu (2) también caracterizó la solubilidad sólida del $\mathrm{MnO}_{2}$ en el $\mathrm{C}_{2} \mathrm{~S}$, según la fórmula:
There is controversy about which $C_{3} S$ polymorph is stabilized by manganese. Some authors (4) state that it is the triclinic phase, although others $(2,3)$ have reported the monoclinic phase.

Esculescu (2) also characterized the solid solution on $\mathrm{MnO}_{2}$ is $\mathrm{C}_{2} \mathrm{~S}$ by the formulae:

$$
\left(\mathrm{Ca}_{2-x} \mathrm{Mn}_{x}\right)\left(\mathrm{Si}_{1-x} \mathrm{Mn}_{x}\right) \mathrm{O}_{4-z} \mathrm{z}=x(3-\mathrm{n})
$$

Sin embargo, Sakurai (5) indicó que la única sustitución posible era la de manganeso por silicio.

En relación a la fase $\mathrm{C}_{3} \mathrm{~A}$ Esculescu (2) demostró que la proporción de átomos de $\mathrm{Mn}$ que se pueden incorporar a la estructura del aluminato tricálcico es inferior al $2 \%$ en peso de $\mathrm{MnO}_{2}$; proponiendo la siguiente fórmula de solubilidad:

\section{Nevertheless, Sakurai (5) proposed that the only possible substitution is of silicon by manganese.}

In relation to $C_{3} A$ phase, Esculescu (2) showed that the proportion of $\mathrm{Mn}$ atoms coming to join to the tricalcium aluminate structure is lower than $2 \%$ of $\mathrm{MnO}_{2}$ weight, and provided the following solubility formulae:

$$
\begin{gathered}
\mathrm{Ca}_{3}\left(\mathrm{Al}_{1-x} \mathrm{Mn}_{\mathrm{x}}\right) \mathrm{O}_{6} \\
0 \leq x \leq 0,01 \ldots 0,02
\end{gathered}
$$


En clínkeres normales que contienen fase ferrítica el manganeso presente se incorpora en su mayor parte a dicha fase ferrítica, mientras que lo hace en mucha menor proporción en las fases silicato y aluminato. Akatsu y Maeda (6) indicaron que cuando se incrementa la relación $\mathrm{Mn} / \mathrm{Fe}+\mathrm{Mn}$ en las soluciones sólidas $C_{6} A(F, M n)_{2}, C_{4} A(F, M n) y$ $\mathrm{C}_{6} \mathrm{~A}_{2}(\mathrm{~F}, \mathrm{Mn})$, los parámetros de red de las estructuras ortorrómbicas sufren alteraciones.

T. W. Parker (7) propuso la composición $4 \mathrm{CaO} . \mathrm{Al}_{2} \mathrm{O}_{3}\left(0,4 \mathrm{Fe}_{2} \mathrm{O}_{3} \cdot 0,6 \mathrm{Mn}_{2} \mathrm{O}_{3}\right)$ como límite de la solubilidad sólida en la fase ferrítica. Finalmente, otros autores (3) indicaron que la presencia de $\mathrm{Mn}_{2} \mathrm{O}_{3}$ en la fase intersticial incrementa su cantidad y actividad, pero disminuye su viscosidad.

El objetivo más importante del presente trabajo es determinar y cuantificar el efecto de la sustitución del manganeso en las estructuras cristalinas de las fases del clínker del cemento portland.

\section{TRABAJO EXPERIMENTAL}

Las mezclas fueron preparadas utilizando reactivos de alta pureza, tales como $\mathrm{SiO}_{2}$ (cuarzo), $\mathrm{CaCO}_{3}$ "Analar", $\mathrm{Al}_{2} \mathrm{O}_{3}$ "Merck", $\mathrm{Fe}_{2} \mathrm{O}_{3}$ "Merck" y oxalato de manganeso (II) "BDH".

\section{FASES SILICATO}

Las composiciones preparadas aparecen recogidas en la Tabla 1 .
However, in normal clinkers which contain a ferrite phases it appear that much of the manganese present is incorpored in ferrite phase, rather that silicate and aluminate phases. Akatsu and Naeda (6) showed that when increased the relation $\mathrm{Mn} / \mathrm{Fe}+\mathrm{Mn}$ in $C_{6} A(F, M n)_{2}, C_{4} A(F, M n)$ and $C_{6} A_{2}(F, M n)$ solid solutions, the unit cell parameters of the orthorombic structure change.

T. W. Parker (7) proposed $4 \mathrm{CaO} \cdot \mathrm{Al}_{2} \mathrm{O}_{3}$ $\left(0,4 \mathrm{Fe}_{2} \mathrm{O}_{3}\right.$ 0,6 Mn $\left.\mathrm{Mn}_{2} \mathrm{O}\right)$ as limit of the solid solution in ferrite phase.

Finnaly, others authors (8) indicated that $\mathrm{Mn}_{2} \mathrm{O}_{3}$ is in the interstitial phase increasing its amount and activity, but decreasing its viscosity.

The main aim of this work is to determine and to quantify the effect of manganese substitution upon the crystal structures of portland cement clinker phases.

\section{EXPERIMENTAL}

The batches were prepared from reagent-grades of $\mathrm{SiO}_{2}$ (quartz), "Analar" $\mathrm{CaCO}_{3}$, "merck" $\mathrm{Al}_{2} \mathrm{O}_{3}$ "Merck" $\mathrm{Fe}_{2} \mathrm{O}_{3}$ and $\mathrm{BDH}$ manganese (II) oxalate.

\section{SILICATE PHASES}

The compositions prepared are showed in Table 1 .

\section{TABLA I (TABLE $\quad$ )}

\begin{tabular}{|c|c|}
\hline $\begin{array}{c}\text { Relación } \mathbf{C a} / \mathbf{S i} \\
\text { (Ca/Si ratio) }\end{array}$ & \multicolumn{1}{|c|}{$\begin{array}{c}\text { Moles de manganeso adicionados } \\
\text { (Mols of manganese added) }\end{array}$} \\
\hline 3 & 0,096 (equivalente a $2.2 \%$ de $\mathrm{Mn}$ ) (equivalent to $2.2 \%$ of $\mathrm{Mn}$ ) \\
3 & 0.241 (equivalente a $5.5 \%$ de $\mathrm{Mn}$ ) (equivalent to 5.5 of $\mathrm{Mn}$ ) \\
2 & 0.070 (equivalente a $2.2 \%$ de $\mathrm{Mn}$ ) (equivalent to $2.2 \%$ of $\mathrm{Mn}$ ) \\
2 & 0.176 (equivalente a $5.3 \%$ de $\mathrm{Mn}$ ) (equivalent to $5.3 \%$ of $\mathrm{Mn}$ ) \\
\hline
\end{tabular}

Se pesaron las cantidades correspondientes de los reactivos, y se homogeneizaron en mortero. Las mezclas secas fueron calentadas, en crisoles de platino y en atmosfera de aire, a temperaturas comprendidas entre $1.300^{\circ} \mathrm{y}$ $1.420^{\circ} \mathrm{C}$, durante varios dias. Se realizaron varias moliendas intermedias durante el tratamiento térmico con el objeto de mejorar su homogeneidad.
Weighed quantities were blended in a mortar, dried and fired in Pt crucibles in air atmosphere at temperatures between $1.300^{\circ}$ and $1.420^{\circ} \mathrm{C}$ for several days, with intermediate grinding to increase homogeneity. 


\section{FASES ALUMINATO}

Se prepararon las siguientes composiciones, en donde el manganeso sustituia parcialmente al aluminio:

\section{ALUMINATE PHASES}

The following mixtures, where aluminium was partially substituted by manganese, where prepared:

$$
\begin{gathered}
\mathrm{Ca}_{3} \mathrm{Al}_{1.902} \mathrm{Mn}_{0.098} \mathrm{O}_{6} \\
\text { and } \\
\mathrm{Ca}_{3} \mathrm{Al}_{1.754} \mathrm{Mn}_{0.246} \mathrm{O}_{6}
\end{gathered}
$$

Los tratamientos térmicos a los que fueron sometidas las muestras, se realizaron a temperaturas entre $1.300^{\circ}$ y $1.460^{\circ} \mathrm{C}$, durante periodos de tiempo variable, de 17 a 84 horas; con moliendas intermedias para incrementar la homogeneidad.

\section{FASE FERRITICA}

Las composiciones con $\mathrm{Mn}$-sustituido fueron preparadas considerando las fórmulas:
Thermal treatments under which were subject the sample were carried out at temperature range between $1.300^{\circ}$ and $1.460^{\circ} \mathrm{C}$, for periods of variable length time ranging between 17 to 84 hours; with intermediate grinding to increase homogeneity.

\section{FERRITE PHASES}

The Mn-substituted solid solutions were prepared assuming the formula:

$$
\mathrm{Ca}_{2} \mathrm{Fe}_{2-x} \mathrm{Mn}_{x} \mathrm{O}_{5}
$$

siendo

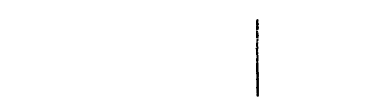

$$
x=0,06,0,17,0,34,0,50 \text { y } 0,64
$$

$$
\mathrm{Ca}_{4} \mathrm{Fe}_{2-x} \mathrm{Mn}_{x} \mathrm{Al}_{2} \mathrm{O}_{10}
$$

siendo

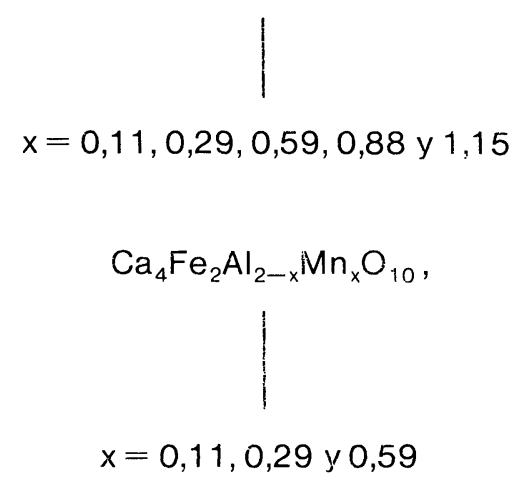

Las cantidades correspondientes de los reactivos fueron homogeneizadas y calentadas, en atmosfera de aire, a temperaturas seleccionadas entre $1.050^{\circ}$ y $1.350^{\circ} \mathrm{C}$, durante varios días y con moliendas intermedias. Se tuvo especial cuidado en la selección de las temperaturas para evitar la formación de fusiones parciales; ya que como es sabido los puntos de fusión de los ferritos disminuyen al aumentar los valores de $X$.

Todas las muestras obtenidas fueron
Weight quantities were blended and fired an air atmosphere at selected temperatures between $1.050^{\circ} \mathrm{C}$ and $1.300^{\circ}$ for several days, with intermediate grinding. Care was taken to avoid partial melting of the batches, as melting points of the ferrites are known to decrease with increasing values of $x$.

All sintered powders were analyzed by $X$-ray 
analizadas por difracción de rayos $X$. Algunas de ellas fueron examinadas por microanálisis de energías dispersivas.

\section{RESULTADOS}

\section{FASES SILICATO}

\section{Difracción de rayos $X$}

Los resultados obtenidos al analizar las composiciones estudiadas por DRX aparecen recogidas en la Tabla Ii. diffraction. Several preparations were

examined by analytical electron microscopy.

\section{RESULTS}

\section{SILICATE PHASES}

\section{X-ray diffraction}

Results of XRD analysis of the studied compositions are showed en Table II.

TABLA II (TABLE II)

Resultado de difracción de rayos $X$ de las fases silicato con $M n$ en disolución sólida (X-Ray powder data for Mn-silicates phases solid solution)

\begin{tabular}{|c|c|c|c|}
\hline $\begin{array}{l}\text { Relación } \mathrm{Ca} / \mathrm{Si} \\
\text { (Ca/Si ratio) }\end{array}$ & $\begin{array}{l}\text { moles } \mathrm{Mn} \text { adicionados } \\
\text { (mol } \mathrm{Mn} \text { added) }\end{array}$ & $\begin{array}{c}\text { Fases } \\
\text { (Phases) }\end{array}$ & \\
\hline 3 & 0.096 & $\begin{array}{l}\mathrm{C}_{3} \mathrm{~S} \text { (Monoclínico) (Monoclinic) } \\
2 \mathrm{CaO} \mathrm{MnO}_{2} \\
\mathrm{\beta C}_{2} \mathrm{~S} \\
\mathrm{CaO}\end{array}$ & $\begin{array}{l}+++ \\
+ \\
+ \\
+\end{array}$ \\
\hline 3 & 0.241 & $\begin{array}{l}\mathrm{C}_{3} \mathrm{~S} \text { (Monoclínico) (Monoclinic) } \\
2 \mathrm{CaO} \mathrm{MnO}_{2} \\
\beta \mathrm{C}_{2} \mathrm{~S} \\
\mathrm{CaO}\end{array}$ & $\begin{array}{l}++ \\
++ \\
+ \\
+\end{array}$ \\
\hline 2 & 0.070 & $\begin{array}{l}\beta \mathrm{C}_{2} \mathrm{~S} \\
\mathrm{CaO} \mathrm{MnO}_{2} \\
\mathrm{CaO}\end{array}$ & $\begin{array}{l}+++ \\
+ \\
0\end{array}$ \\
\hline 2 & 0.176 & $\begin{array}{l}\beta \mathrm{C}_{2} \mathrm{~S} \\
\mathrm{CaO} \mathrm{MnO}_{2} \\
\mathrm{CaO}\end{array}$ & $\begin{array}{l}+++ \\
++ \\
0\end{array}$ \\
\hline
\end{tabular}

$+++=$ Abundante (Abundant); $++=$ Moderado (Moderate) $++=$ Poco (little); $0=$ Trazas $($ Trace $)$

\section{Microanálisis por espectroscopia de energías dispersivas}

Las muestras analizadas por esta técnica fueron las siguientes: $\mathrm{Ca} / \mathrm{Si}=3$ con 0,241 moles de Mn obtenida al calentar la mezcla a $1.420^{\circ} \mathrm{C}$ durante 32 horas; y $\mathrm{Ca} / \mathrm{Si}=2$ con 0,176 moles de $\mathrm{Mn}$ obtenida por calentamiento a $1.420^{\circ} \mathrm{C}$ durante 38 horas.

Los análisis fueron realizados sobre 10 cristales diferentes de $\mathrm{C}_{3} \mathrm{~S}$ y $\mathrm{C}_{2} \mathrm{~S}$ en ambas composiciones. Los resultados de los citados análisis aparecen resumidos en la Tabla III.

\section{Analytical electron microscopy}

The following samples were analyzed by this technique:

$\mathrm{Ca} / \mathrm{Si}=3$ with $0,241 \mathrm{mo} / \mathrm{s}$ of Mn obtained by heating at $1.420^{\circ} \mathrm{C}$ for 32 hours, and $\mathrm{Ca} / \mathrm{Si}=2$ with $0,176 \mathrm{mo} / \mathrm{s}$ of $\mathrm{Mn}$, obtained by heating at $1.420^{\circ} \mathrm{C}$ for 38 hours.

The analyses were made on 10 different crystals, selected to be $C_{3} S$ and $C_{2} S$, from each batch. The results from these analysis are summarized in Table III. 
TABLA III (TABLE III)

Resultados del microanálisis en las fases silicato con Mn en disolución sólida (Analytical electron microscopy on sample of Mn-silicate phases)

\begin{tabular}{|c|c|c|c|c|}
\hline $\begin{array}{c}\text { Relación } \mathbf{C a} / \mathbf{S i} \\
\text { (Ca/Si ratio) }\end{array}$ & $\begin{array}{c}\mathbf{S i} / \mathrm{Ca} \\
\text { (Si/Ca) }\end{array}$ & $\begin{array}{c}\mathbf{M n} / \mathrm{Ca} \\
\text { (Mn/Ca) }\end{array}$ & $\begin{array}{c}\text { Fórmulas propuestas } \\
\text { (Proposed formulae) }\end{array}$ & $\begin{array}{c}\text { \% Mn solubilizado } \\
\text { (\% Mn solid solution) }\end{array}$ \\
\hline 3 & 0.318 & 0.012 & $\mathrm{Ca}_{3}\left(\mathrm{Si}_{0.954} \mathrm{Mn}_{0.036}\right) \mathrm{O}_{5}$ & $0.72 \pm 0.11$ \\
2 & 0.473 & 0.024 & $\mathrm{Ca}_{2}\left(\mathrm{Si}_{0.946} \mathrm{Mn}_{0.048}\right) \mathrm{O}_{4}$ & $1.53 \pm 0.12$ \\
\hline
\end{tabular}

\section{FASES ALUMINATO}

\section{Difracción de rayos $X$}

Los resultados de los análisis realizados por DRX en las composiciones estudiadas se muestran en la Tabla IV. Según se desprende de la Tabla, el $\mathrm{C}_{3} \mathrm{~A}$ en su forma cúbica, es la fase mayoritaria en ambas mezclas.

\section{ALUMINATE PHASES}

\section{X-ray diffraction}

Results of XRD analysis of the studied compositions are showed on Table IV.

$$
\text { TABLA IV (TABLE IV) }
$$

Resultados de difracción de rayos $X$ de las soluciones sólidas $\mathrm{Ca}_{3} \mathrm{Al}_{2-{ }_{x}} \mathrm{Mn}_{\mathrm{x}} \mathrm{O}_{6}$ ( $X$-ray power data for $\mathrm{Ca}_{3} \mathrm{Al}_{2-x} \mathrm{Mn}_{x} \mathrm{O}_{6}$ solid solutions)

\begin{tabular}{|c|c|c|c|}
\hline $\begin{array}{l}\text { Composición } \\
\text { (Composition) }\end{array}$ & $\begin{array}{c}x \\
(x)\end{array}$ & $\begin{array}{l}\text { Fases } \\
\text { (Phases) }\end{array}$ & \\
\hline \multirow{2}{*}{$\mathrm{Ca}_{3} \mathrm{Al}_{2-x} \mathrm{Mn}_{x} \mathrm{O}_{6}$} & 0.098 & $\begin{array}{l}\mathrm{C}_{3} \mathrm{~A} \text { (cúbico) (cubic) } \\
\mathrm{Ca}_{2} \mathrm{AlMnO}_{5} \\
\mathrm{CaO}\end{array}$ & $\begin{array}{l}+++ \\
+ \\
0\end{array}$ \\
\hline & 0.246 & $\begin{array}{l}\mathrm{C}_{3} \mathrm{~A} \text { (cúbico) (cubic) } \\
\mathrm{Ca}_{2} \mathrm{AlMnO}_{5} \\
\mathrm{CaO}\end{array}$ & $\begin{array}{l}+++ \\
++ \\
+\end{array}$ \\
\hline
\end{tabular}

El contenido de la fase $\mathrm{Ca}_{2} \mathrm{AlMnO}_{5}$ (identificado por primera vez por Sakurai (5), 1960) se ve incrementado al aumentar el porcentaje de Mn presente.

\section{Microanálisis por espectroscipia de energías dispersivas}

La muestra analizada por esta técnica fue: $\mathrm{Ca}_{3} \mathrm{~A}_{1,754} \mathrm{Mn}_{0,246} \mathrm{O}_{6}$ que fue obtenida por calentamiento a $1.420^{\circ} \mathrm{C}$ durante 84 horas. Los resultados obtenidos aparecen en la Tabla V.
According to this Table, cubic $C_{3} A$ is the most frequent phase for both mixtures. $\mathrm{Ca}_{2} \mathrm{AlMnO}_{5}$ (first idenfied by Sukarai (5), 1960) increases in quantity when the porcentage of $\mathrm{Mn}$ increases.

\section{Analytical electron microscopy}

The sample analyzed by this technique was $\mathrm{Ca}_{3} \mathrm{Al}_{1.754} \mathrm{Mn}_{0.246} \mathrm{O}_{6}$ and was obtained by heating at $1.420^{\circ} \mathrm{C}$ for 84 hours. The results are showed in Table $\mathrm{V}$.

$$
\text { TABla } V \quad \text { (TABLE V) }
$$

Resultados del microanálisis en $\mathrm{Ca}_{3} \mathrm{Al}_{2-x} \mathrm{Mn}_{\mathrm{x}} \mathrm{O}_{6}$ (Analytical electrom microscopy on $\mathrm{Ca}_{3} \mathrm{Al}_{2-x} \mathrm{Mn}_{x} \mathrm{O}_{6}$ )

\begin{tabular}{|c|c|c|c|}
\hline $\begin{array}{c}\text { Al/Ca } \\
\text { (Al/Ca) }\end{array}$ & $\begin{array}{c}\text { Mn/Ca } \\
(\mathrm{Mn} / \mathrm{Ca})\end{array}$ & $\begin{array}{c}\text { Formula propuesta } \\
\text { (Proposed formula) }\end{array}$ & $\begin{array}{c}\% \text { Mn solubilizado } \\
\text { (\% Mn solid solution) }\end{array}$ \\
\hline 0.663 & 0.013 & $\left(\mathrm{Ca}_{2.984} \mathrm{Mn}_{0.016}\right)\left(\mathrm{Al}_{1.979} \mathrm{Mn}_{0.021}\right) \mathrm{O}_{6}$ & $0.78 \pm 0.12$ \\
\hline
\end{tabular}




\section{FASE FERPITICA}

\section{Difracción de rayos $x$}

En la Tabla VI se muestran los resultados obtenidos tras el análisis por DRX de las composiciones $\mathrm{Ca}_{2} \mathrm{Fe}_{2-x} \mathrm{Mn}_{x} \mathrm{O}_{5}$.

\section{FERRITE PHASES}

\section{X-ray diffraction}

Results of the XRD analysis of $\mathrm{Ca}_{2} \mathrm{Fe}_{2 \cdot x} \mathrm{Mn}_{x} \mathrm{O}_{5}$ compositions are showed on Table V!.

TABLA VI (TABLE VI)

Resultados de difracción de rayos $X$ de las soluciones sólidas $\mathrm{Ca}_{2} \mathrm{Fe}_{2-x} \mathrm{Mn}_{x} \mathrm{O}_{5}$ (X-ray powder data for $\mathrm{Ca}_{2} \mathrm{Fe}_{2-x} \mathrm{Mn}_{x} \mathrm{O}_{5}$ solid solutions)

\begin{tabular}{|c|c|c|c|}
\hline $\begin{array}{l}\text { Composicion } \\
\text { (Composition) }\end{array}$ & $\stackrel{x}{(x)}$ & \multicolumn{2}{|c|}{$\begin{array}{c}\text { Fases } \\
\text { (Phases) }\end{array}$} \\
\hline \multirow{5}{*}{$\mathrm{Ca}_{2} \mathrm{Fe}_{2-\mathrm{x}} \mathrm{Mn}_{\mathrm{x}} \mathrm{O}_{5}$} & 0.06 & $\mathrm{C}_{2} \mathrm{~F}_{\mathrm{ss}}$ & +++ \\
\hline & 0.17 & $\mathrm{C}_{2} \mathrm{~F}_{\mathrm{ss}}$ & +++ \\
\hline & 0.34 & $\begin{array}{l}\mathrm{C}_{2} \mathrm{~F}_{\mathrm{ss}} \\
\mathrm{CaO}\end{array}$ & $\begin{array}{l}+++ \\
0\end{array}$ \\
\hline & 0.50 & $\begin{array}{l}\mathrm{C}_{2} \mathrm{~F}_{\mathrm{ss}} \\
\mathrm{CaO}\end{array}$ & $\begin{array}{l}+++ \\
++\end{array}$ \\
\hline & 0.64 & $\begin{array}{l}\mathrm{C}_{2} \mathrm{~F}_{\mathrm{ss}} \\
\mathrm{CaO} \\
2 \mathrm{CaOH}\end{array}$ & $\begin{array}{l}++ \\
+++ \\
++\end{array}$ \\
\hline
\end{tabular}

Cuando se incrementa el contenido de $\mathrm{Mn}$ se observa una alteración en la posición de algunas reflexiones. Muchas de éstas van adoptando progresivamente formas más anchas y difusas. Este proceso parece concluir, en base a los resultados obtenidos por difracción de rayos $\mathrm{X}$, con $6,87 \%$ en peso de $\operatorname{Mn}(x=0,34)$.

Los resultados de DRX de las composiciones $\mathrm{Ca}_{4} \mathrm{Fe}_{2-x} \mathrm{Mn}_{x} \mathrm{Al}_{2} \mathrm{O}_{10}$ y $\mathrm{Ca}_{4} \mathrm{Fe}_{2} \mathrm{Al}_{2-x} \mathrm{Mn}_{\mathrm{x}} \mathrm{O}_{10}$ se muestran resumidos en la Tabla VII.

En ambas composiciones, cuando los contenidos en $\mathrm{Mn}$ son inferiores a 6,67 en peso $\%(x=0,59)$, la única fase presente es un $\mathrm{C}_{4} \mathrm{AF}$ ss tipo disolución sólida. Por encima de $6,67 \%$, se detecta la presencia de otras fases de $\mathrm{Mn}$.

\section{Microanálisis por espectroscopia de energías dispersivas}

Las muestras analizadas por esta técnica fueron:
Several changes were observed in the positions of reflections in the powder patterns with increasing $\mathrm{Mn}$ content; many of the reflections become progressively broadered and more diffuse. This process appears, on the basis of $X$-ray results, to have been completed by $6,87 \%$ wt $M n(x=0,34)$.

Results of the XRD analysis of $\mathrm{Ca}_{4} \mathrm{Fe}_{2 \cdot x} \mathrm{Mn}_{x} \mathrm{Al}_{2} \mathrm{O}_{10}$ and $\mathrm{Ca}_{4} \mathrm{Fe}_{2} \mathrm{Al}_{2 \cdot x} \mathrm{Mn}_{x} \mathrm{O}_{10}$ compositions are summarized in Table VII.

Up to $6,67 \% M n(x=0,59)$ in both compositions the amain phase present is a $C_{4} A F$ type solid solution, above $6,67 \mathrm{w} \%$ others $M n$ phases are detected.

\section{Analytical electron microscopy}

The samples analyzed by this technique were:

y

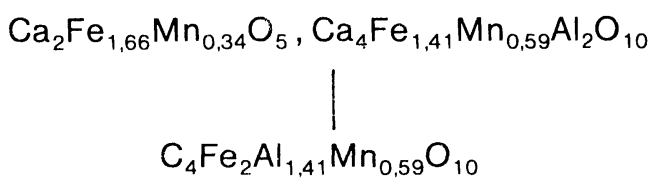


Los resultados obtenidos aparecen en la Tabla VIII.
Los results obtained are showed in Table VIII.

TABLA VII (TABLE VII)

Resultados de difracción de rayos $X$ de las soluciones sólidas $\mathrm{Ca}_{4} \mathrm{Fe}_{2-\mathrm{x}} \mathrm{Mn}_{\mathrm{x}} \mathrm{Al}_{2} \mathrm{O}_{10}$

$$
\text { y } \mathrm{Ca}_{4} \mathrm{Fe}_{2} \mathrm{Al}_{2-x} \mathrm{Mn}_{\mathrm{x}} \mathrm{O}_{10}
$$

(X-ray powder data for $\mathrm{Ca}_{4} \mathrm{Fe}_{2-x} \mathrm{Mn}_{x} \mathrm{Al}_{2} \mathrm{O}_{10}$ and $\mathrm{Ca}_{4} \mathrm{Fe}_{2} \mathrm{Al}_{2-x} \mathrm{Mn}_{x} \mathrm{O}_{10}$ solid solutions)

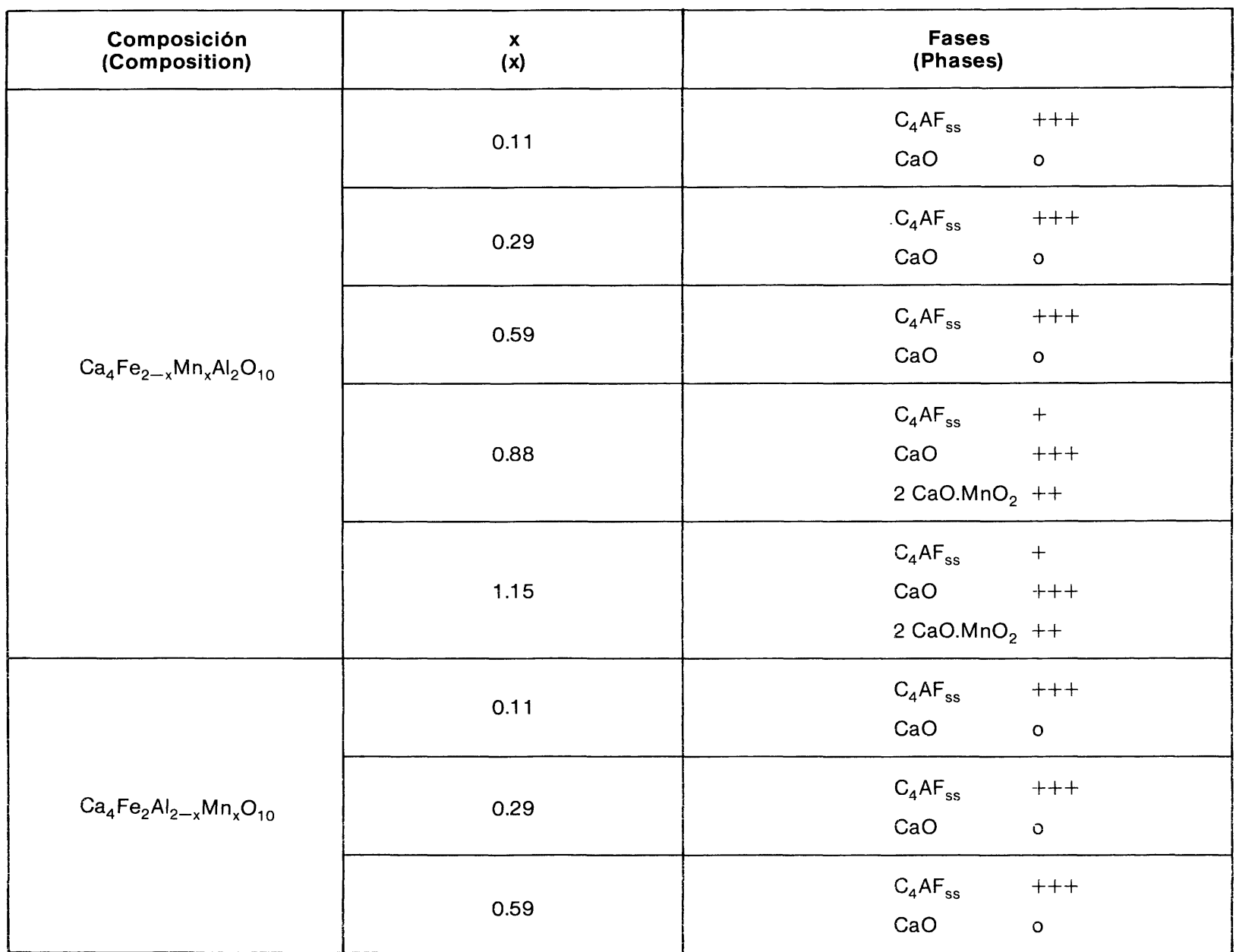

TABLA VIII (TABLE VIII)

Resultados del microanálisis en $\mathrm{Ca}_{2} \mathrm{Fe}_{1.66} \mathrm{Mn}_{0.34} \mathrm{O}_{5}, \mathrm{Ca}_{4} \mathrm{Fe}_{1.44} \mathrm{Mn}_{0.59} \mathrm{O}_{10}$ y $\mathrm{Ca}_{4} \mathrm{Fe}_{2} \mathrm{Al}_{1.41} \mathrm{Mn}_{0.59} \mathrm{O}_{10}$ disoluciones sólidas

(Analytical electron microscopy on $\mathrm{Ca}_{2} \mathrm{Fe}_{1.66} \mathrm{Mn}_{0.34} \mathrm{O}_{5}, \mathrm{Ca}_{4} \mathrm{Fe}_{1.44} \mathrm{Mn}_{0.59} \mathrm{O}_{10}$ and $\mathrm{Ca}_{4} \mathrm{Fe}_{2} \mathrm{Al}_{1.4} \mathrm{Mn}_{0.59} \mathrm{O}_{10}$ solid solutions)

\begin{tabular}{|c|c|c|c|c|}
\hline $\begin{array}{c}\mathrm{Fe} / \mathrm{Ca} \\
(\mathrm{Fe} / \mathrm{Ca})\end{array}$ & $\begin{array}{c}\mathrm{Al} / \mathrm{Ca} \\
(\mathbf{A l} / \mathrm{Ca})\end{array}$ & $\begin{array}{c}\mathrm{Mn} / \mathrm{Ca} \\
(\mathrm{Mn} / \mathrm{Ca})\end{array}$ & $\begin{array}{c}\text { Formulas prupuestas } \\
\text { (Proposed formulae) }\end{array}$ & $\begin{array}{c}\% \text { Mn solubilizado } \\
\text { (\% Mn solid solution) }\end{array}$ \\
\hline 0.861 & - & 0.171 & $\left(\mathrm{Ca}_{1.969} \mathrm{Mn}_{0.031}\right)\left(\mathrm{Fe}_{1.696} \mathrm{Mn}_{0.304}\right) \mathrm{O}_{5}$ & $6.80 \pm 0.87$ \\
0.420 & 0.445 & 0.150 & $\left(\mathrm{Ca}_{3.970} \mathrm{Mn}_{0.030}\right)\left(\mathrm{Fe}_{1.668} \mathrm{Mn}_{0.332}\right)\left(\mathrm{Al}_{1.767} \mathrm{Mn}_{0.233}\right) \mathrm{O}_{10}$ & $6.73 \pm 2.51$ \\
0.428 & 0.458 & 0.154 & $\left(\mathrm{Ca}_{3.922} \mathrm{Mn}_{0.078}\right)\left(\mathrm{Fe}_{1.679} \mathrm{Mn}_{0.321}\right)\left(\mathrm{Al}_{1.797} \mathrm{Mn}_{203}\right) \mathrm{O}_{10}$ & $6.82 \pm 0.31$ \\
\hline
\end{tabular}




\section{DISCUSION}

La presencia de manganeso favorece la formación de silicato tricálcico en su forma monoclínica. Sólo es posible una sustitución limitada del $\mathrm{Mn}$ en el $\mathrm{C}_{3} \mathrm{~S}$, ya que excesos de este elemento provocan la formación de $2 \mathrm{CaOMnO}_{2}$. Como se podría esperar el contenido de esta fase se ve incrementada al aumentar la cantidad de $\mathrm{Mn}$ adicionado.

En las muestras con relación $\mathrm{Ca} / \mathrm{Si}=2$, la presencia de manganeso origina la formación de $\mathrm{CaO} . \mathrm{MnO}_{2}$.

Esta diferencia en el compuesto de manganeso formado está motivada por la mayor relación $\mathrm{CaO} / \mathrm{MnO}_{2}$ en la primera de las composiciones, lo que conlleva que se favorezca la formación de fases más ricas en $\mathrm{CaO}$.

Las fórmulas de solubilidad propuestas, calculadas en base a los cinco o cuatro oxigenos disponibles, y utilizando las relaciones $\mathrm{Ca} / \mathrm{Mn}$ y $\mathrm{Ca} / \mathrm{Si}$ obtenidos a partir de microanálisis por espectroscopía de energías dispersivas son:

\section{DISCUSSION}

Manganese doping promotes formation of monoclinic tricalcium silicate. Only limited substitution of $M n$ into $\mathrm{C}_{3} \mathrm{~S}$ is possible and the excess of $\mathrm{Mn}$ appears as $2 \mathrm{CaO} \cdot \mathrm{MnO}_{2}$. As expected, the content of this phase increases on the amount of Mn increases too.

In the manganese subtituted sample at the $\mathrm{Ca} / \mathrm{Si}=2$ ratio, the manganese compound, $\mathrm{CaO} . \mathrm{MnO}_{2}$, are developed. These differences are due the higher $\mathrm{CaO} / \mathrm{MnO}_{2}$ ratio in the first composition which favours formation of more $\mathrm{CaO}$ rich accesory phases.

The proposed solid solution formula, recalculated on the basis of five or four oxygens, and using $\mathrm{Ca} / \mathrm{Mn}$ and $\mathrm{Ca} / \mathrm{Si}$ ratios from analytical electron microscopy are:

y

$$
\begin{gathered}
\mathrm{Ca}_{3}\left(\mathrm{Si}_{0,954} \mathrm{Mn}_{0,035}\right) \mathrm{O}_{5} \\
1 \\
\mathrm{Ca}_{2}\left(\mathrm{Si}_{0.946} \mathrm{Mn}_{0.048}\right) \mathrm{O}_{4}
\end{gathered}
$$

Según estas fórmulas, en

ambas composiciones $\left(\mathrm{C}_{3} \mathrm{~S}\right.$ y $\left.\mathrm{C}_{2} \mathrm{~S}\right)$ todo el manganeso presente está como $\mathrm{Mn}^{4+}$ ocupando posiciones de $\mathrm{Si}^{4+}$. Los radios ionicos del $\mathrm{Mn}^{4+}$ y $\mathrm{Si}^{4+}$ son apreciablemente distintos 0,60 y $0,42 \AA$, respectivamente. Esta diferencia limita severamente una sustitución a gran escala del $\mathrm{Mn}^{4+}$ por $\mathrm{Si}^{4+}(\%$ de $\mathrm{Mn}$ solubilizado en $\mathrm{C}_{3} \mathrm{~S}$ y $\mathrm{C}_{2} \mathrm{~S}$ es de 0,72 $\pm 0,11$ y $1,53 \pm 0,12$, respectivamente)

El estudio realizado a los compuestos que responden a la fórmula general $\mathrm{Ca}_{3} \mathrm{Al}_{2-x} \mathrm{Mn}_{\mathrm{x}} \mathrm{O}_{6}$ permite extraer interpretaciones concluyentes sobre el efecto que ejerce el $\mathrm{Mn}$ en la estructura del $C_{3} A$.

Los resultados obtenidos por DRX muestran que el $\mathrm{C}_{3} \mathrm{~A}$ estabilizado se encuentra en la forma cúbica. Por otra parte, la presencia de elevados contenidos de $\mathrm{Mn}$ favorece la formación de $\mathrm{Ca}_{2} \mathrm{AIMnO}_{5}$, compuesto que precisa cantidades importantes de $\mathrm{CaO}$ y $\mathrm{Al}_{2} \mathrm{O}_{3}$, lo que provoca una disminución en el contenido de $\mathrm{C}_{3} \mathrm{~A}$. Cantidades muy elevadas de $\mathrm{Mn}$ pueden llegar incluso a inhibir la
According to these formula, in both compositions $\left(\mathrm{C}_{3} \mathrm{~S}\right.$ and $\left.\mathrm{C}_{2} \mathrm{~S}\right)$ all manganese is present as $\mathrm{Mn}^{4+}$ occupying $\mathrm{Si}^{4+}$ positions. The ionic sizes of $\mathrm{Mn}^{4+}$ and $\mathrm{Si}^{4+}$ are noticeably different, 0,60 and 0,42 $\AA$, respectively, and this difference severely limits the maximum extent of substitution of $\mathrm{Si}^{4+}$ by $\mathrm{Mn}^{4+}(\% \mathrm{Mn}$ solid solution into $C_{3} S$ and $C_{2} S$ are $0,72 \pm 0,11$ and $1,53 \pm 0,12$, respectively).

The study carried out for compounds attending to the general formula $\mathrm{Ca}_{3} \mathrm{Al}_{2 \cdot x} \mathrm{Mn}_{\mathrm{x}} \mathrm{O}_{6}$ allows induce several conclusive interpretations about effects exerted by $\mathrm{Mn}$ on $\mathrm{C}_{3}$ A structure. Results obtained by XRD show that stabilized $C_{3} A$ is cubic on the other hand, the presence of high quantities of $\mathrm{Mn}$ favours the formation of $\mathrm{Ca}_{2} \mathrm{AlMnO}_{5}$, compound which need s for its formation a very important amount of $\mathrm{CaO}$ and $\mathrm{Al}_{2} \mathrm{O}_{3}$, which produces a decrease in $\mathrm{C}_{3} \mathrm{~A}$ contents. Suitably high quantities of $M n$ inhibit a $C_{3}$ A formation resulting in its formation $\mathrm{Ca}_{2} \mathrm{AlMnO}_{5}$ (9). 
formación de $\mathrm{C}_{3} \mathrm{~A}$, por favorecerse el desarrollo de $\mathrm{Ca}_{2} \mathrm{AlMnO}_{5}$ (9).

El límite de solubilidad sólida del Mn en la estructura del $\mathrm{C}_{3} \mathrm{~A}$ fue determinada por microanálisis de energías dispersivas, y tiene una valor de 0,78 $\pm 0,12 \%$ en peso. La fórmula de solubilidad sólida propuesta es:
The limit of Mn solid solubility in the $C_{3} A$ structure was determined by analytical electron microscopy and has a value of 0,78 $\pm 0,12$ (\% $w t)$. The solubility formulae proposed in this work is:

$$
\left(\mathrm{Ca}_{2,984} \mathrm{Mn}_{0.016}\right)\left(\mathrm{Al}_{1,979} \mathrm{Mn}_{0.021}\right) \mathrm{O}_{6}
$$

Según esta fórmula, el manganeso se encuentra aproximadamente en un $43 \%$ como $\mathrm{Mn}^{2+}$, y en un $57 \%$ como $\mathrm{Mn}^{3+}$. Los radios iónicos del $\mathrm{Mn}^{2+}$ y del $\mathrm{Ca}^{2+}$ son muy similares (0,91 y 0,99 $\AA$ respectivamente), lo que permite que el $\mathrm{Ca}^{2+}$ puede ser sustituido por $\mathrm{Mn}^{2+}$.

La diferencia de los radios iónicos del $\mathrm{Mn}^{3+}$ y $\mathrm{Al}^{3+}(0,66$ y $0,51 \AA$, respectivamente) provoca que la sustitución en las posiciones tetraétricas del $\mathrm{Al}^{3+}$ sea limitada, concretamente con un valor de 0,021 moles de $\mathrm{Mn}$. El estado de oxidación +3 es el que está inicialmente favorecido por las condiciones oxidantes del tratamiento térmico.

La fórmula de solubilidad propuesta por Esculescu (2) es la siguiente:
According to this formulae, manganese is approximately found at percentages of $43 \%$ for $\mathrm{Mn}^{2+}$ and at $57 \%$ for $\mathrm{Mn}^{3+}$. The similarity between $\mathrm{Mn}^{2+}$ and $\mathrm{Ca}^{2+}$ ion radii $(0,91$ and 0,99 $A$, respectively) allows the $\mathrm{Ca}^{2+}$ be substituted by $\mathrm{Mn}^{2+}$.

$\mathrm{Mn}^{3+}$ and $\mathrm{Al}^{3+}$ ion radii $(0,66$ and $0,51 \mathrm{~A}$ respectively) allow a limited substitution for $\mathrm{Al}^{3+}$ tetrahedrical positions, with a value of $0,021 \mathrm{~mol}$ of $\mathrm{Mn}$. The +3 oxidation state is initially favoured by thermal treatment under oxidizing conditions.

The solubility formulae proposed by Esculescu (2) is as follow:

$$
\mathrm{Ca}_{3}\left(\mathrm{Al}_{1-x} \mathrm{Mn}_{x}\right) \mathrm{O}_{6} \mathrm{O} \leq 0,01 \ldots 0,02
$$

Según esta fórmula, el límite de sustitución de $\mathrm{Al}^{3+}$ por $\mathrm{Mn}^{3+}$ es de 0,02 moles de $\mathrm{Mn}$. Este valor es prácticamente idéntico al obtenido en este trabajo $(0,021$ moles de $\mathrm{Mn})$. Sin embargo, Esculescu no consideró la posible sustitución de $\mathrm{Ca}^{2+}$ por $\mathrm{Mn}^{2+}$, que ha sido confirmada en el presente trabajo.

A partir de los resultados obtenidos es posible dar una explicación consistente de los efectos provocados por la incorporación del manganeso en las estructuras de las fases ferríticas.

Los resultados obtenidos por microanálisis demuestran que en las composiciones $\mathrm{Ca}_{2} \mathrm{Fe}_{2-x} \mathrm{Mn}_{\mathrm{x}} \mathrm{O}_{5}$, el límite en la disolución sólida del manganeso en el $\mathrm{C}_{2} \mathrm{~F}$ está alrededor de un $6,80 \pm 0,87 \%$, valor que coincide plenamente al obtenido por difracción de rayos $X(6,87 \%)$. Se puede, pues concluir que la solubilidad sólida del manganeso es el $\mathrm{C}_{2} \mathrm{~F}$, a temperaturas subsolidus y en condiciones oxidantes, es del orden del 6,7\% en peso de Mn.

La fórmula de solubilidad propuesta en este trabajo es:
According to this formulae, the limit of $\mathrm{Mn}^{3+}$ substitution is $0,02 \mathrm{~mol}$ of $\mathrm{Mn}$. This value is practically identical to the are obtained in this work $(0,021 \mathrm{~mol}$ of $\mathrm{Mn})$. Esculescu, however, did not take into account the possible substitution of $\mathrm{Ca}^{2+}$ by $\mathrm{Mn}^{2+}$ which has been observed in this work.

From the data obtained, a consistent explanation of the effects of introducing manganese into the lattice of the ferrite phases can be inferred. In $\mathrm{Ca}_{2} \mathrm{Fe}_{2-x} \mathrm{Mn}_{x} \mathrm{O}_{5}$ solid solutions by analytical electron microscopy is showed that the limit of solid solution of $\mathrm{Mn}$ in $\mathrm{C}_{2} \mathrm{~F}$ is about 6,80 $\pm 0,87$ (wt \%), this value being very close to the one obtained by $X$-ray diffraction $(6,87 w t \%)$, it is concluded that the limit of solid solution of manganese in $\mathrm{C}_{2} \mathrm{~F}$ close to solidus temperatures and in oxidizing atmosphere lies in the range 6-7 wt \% $M n$.

The solubility formulae proposed in this work is: 


$$
\left(\mathrm{Ca}_{1,969} \mathrm{Mn}_{0,031}\right)\left(\mathrm{Fe}_{1,969} \mathrm{Mn}_{0,304}\right) \mathrm{O}_{5}
$$

Según se desprende de esta fórmula, el manganeso está presente como $\mathrm{Mn}^{2+}$ y $\mathrm{Mn}^{3+}$. La similitud de los tamaños iónicos del $\mathrm{Fe}^{3+}$ y el $\mathrm{Mn}^{3+}(0,66$ y $0,64 \AA$, respectivamente) permite una amplia incorporación de $\mathrm{Mn}^{3+}$ en las posiciones del $\mathrm{Fe}^{3+}$. El ion $\mathrm{Mn}^{3+}$ es la especie favorecida por las condiciones oxidantes del tratamiento térmico, aunque se ha detectado una pequeña cantidad de $\mathrm{Mn}^{2+}$ ocupando posiciones de $\mathrm{Ca}^{2+}$.

En las composiciones $\mathrm{Ca}_{4} \mathrm{Fe}_{2-x} \mathrm{Mn}_{\mathrm{x}} \mathrm{Al}_{2} \mathrm{O}_{10} \mathrm{y}$ $\mathrm{Ca}_{4} \mathrm{Fe}_{2} \mathrm{Al}_{2-x} \mathrm{Mn}_{\mathrm{x}} \mathrm{O}_{10}$ se ha encontrado por microanálisis y difracción de rayos $X$ que el límite de disolución sólida del $\mathrm{Mn}$ en el $\mathrm{C}_{4} \mathrm{AF}$ está próximo a 6,6 - 6,9\% en peso. Ambas disoluciones sólidas $\mathrm{Ca}_{4} \mathrm{Fe}_{2-x} \mathrm{Mn}_{x} \mathrm{Al}_{2} \mathrm{O}_{10} \mathrm{y}$ $\mathrm{Ca}_{4} \mathrm{Fe}_{2} \mathrm{Al}_{2-x} \mathrm{Mn}_{\mathrm{x}} \mathrm{O}_{10}$ muestran valores similares de $\%$ de Mn solubilizado es decir, 6,73 $\pm 2,51$ y $6,82 \pm 0,31 \%$ en peso, respectivamente.

Las fórmulas propuestas son:
According to this formulae, manganese is present as $\mathrm{Mn}^{2+}$ and $\mathrm{Mn}^{3+}$. The ionic sizes of $\mathrm{Mn}^{3+}$ and $\mathrm{Fe}^{3+}$ are certainly very close $(0,66$ and 0,64 A, respectively). This similarity between $\mathrm{Mn}^{3+}$ and $\mathrm{Fe}^{3+}$ ion radii allows a extent substitution of $\mathrm{Fe}^{3+}$ by $\mathrm{Mn}^{3+}$. This ion, $\mathrm{Mn}^{3+}$, is favoured by thermal treatment under oxidizing conditions. However, it was detected a little amount of $\mathrm{Mn}^{2+}$ occupying $\mathrm{Ca}^{2+}$ positions.

In $\mathrm{Ca}_{4} \mathrm{Fe}_{2-x} \mathrm{Mn}_{x} \mathrm{Al}_{2} \mathrm{O}_{10}$ and $\mathrm{Ca}_{4} \mathrm{Fe}_{2} \mathrm{Al}_{2-x} \mathrm{Mn}_{x} \mathrm{O}_{10}$ solid solutions, it was found by analytical electron microscopy and $X$-ray diffraction that the limit of solid solution of $\mathrm{Mn}$ in $\mathrm{C}_{4} \mathrm{AF}$ is close to $6,6-6,9 w t(\%)$. Both solid solutions,

$\mathrm{Ca}_{4} \mathrm{Fe}_{2-x} \mathrm{Mn}_{x} \mathrm{Al}_{2} \mathrm{O}_{10}$ and $\mathrm{Ca}_{4} \mathrm{Fe}_{2} \mathrm{Al}_{2-x} \mathrm{Mn}_{x} \mathrm{O}_{10}$, have similary values of $\% \mathrm{Mn}$ solid solution,

$6,73 \pm 2,51$ and $6,82 \pm 0,31 w t \%$, respectively

$$
\left(\mathrm{Ca}_{3.970} \mathrm{Mn}_{0,030}\right)\left(\mathrm{Fe}_{1,608} \mathrm{Mn}_{0,332}\right)\left(\mathrm{Al}_{1,767} \mathrm{Mn}_{0,233}\right) \mathrm{O}_{10}
$$

y

$$
\left(\mathrm{Ca}_{3,922} \mathrm{Mn}_{0,078}\right)\left(\mathrm{Fe}_{1,679} \mathrm{Mn}_{0,321}\right)\left(\mathrm{Al}_{1,797} \mathrm{Mn}_{0,203}\right) \mathrm{O}_{10}
$$

Según estas fórmulas, el $\mathrm{Mn}^{3+}$ sustituye tanto al $\mathrm{Fe}^{3+}$ como al $\mathrm{Al}^{3+}$ en la estructura del $\mathrm{C}_{4} \mathrm{AF}$. Una pequeña cantidad de $\mathrm{Mn}^{2+}$ se encuentra sustituyendo al $\mathrm{Ca}^{2 *}$.

Los radios iónicos del $\mathrm{Mn}^{3+}$ y $\mathrm{Fe}^{3+}$ son más similares que los de $\mathrm{Mn}^{3+}$ y $\mathrm{Al}^{3+}\left(\mathrm{r}_{\mathrm{Mn} 3+}=0,66\right.$ $\AA, r_{\mathrm{Fe} 3+}=0,64 \AA$ y $r_{\mathrm{Al} 3+}=0,51 \AA$. Estos valores justifican que la sustitución de $\mathrm{Mn}^{3+}$ por $\mathrm{Fe}^{3+}$ sea superior a la de $\mathrm{Mn}^{3+}$ por $\mathrm{Al}^{3+}$.

Por otra parte, en ambas composiciones hay una pequeña cantidad de $\mathrm{Mn}^{2+}$ que ocupa posiciones de $\mathrm{Ca}^{2+}$. La diferencia en los contenidos de $\mathrm{Ca}^{2+}$ en dichas composiciones $\left(\mathrm{Ca}_{4} \mathrm{Fe}_{2-x} \mathrm{Mn}_{\mathrm{x}} \mathrm{Al}_{2} \mathrm{O}_{10}\right.$ y $\left.\mathrm{Ca}_{4} \mathrm{Fe}_{2} \mathrm{Al}_{2-x} \mathrm{Mn}_{\mathrm{x}} \mathrm{O}_{10}\right)$ es debida muy probablemente a las diferentes condiciones oxidantes de los hornos empleados, ya que fueron utilizados dos hornos distintos de laboratorio.

\section{CONCLUSIONES}

1.- Las solubilidades sólidas del $\mathrm{Mn}$ en las estructuras del $\mathrm{C}_{3} \mathrm{~S}$ y $\mathrm{C}_{2} \mathrm{~S}$ son $0,72 \pm 0,11$ y $1,53 \pm 0,12 \%$ en peso, respectivamente. Las fórmulas propuestas para estas solubilidades son:
According to these formula, $\mathrm{Mn}^{3+}$ is accopying $\mathrm{Fe}^{3+}$ and $\mathrm{Al}^{3+}$ positions into $\mathrm{C}_{4} A \mathrm{~F}$. A little amount of $\mathrm{Ca}^{2+}$ is substituted by $\mathrm{Mn}^{2+}$.

The ionic radii of $\mathrm{Mn}^{3+}$ and $\mathrm{Fe}^{3+}$ are more similar than those of $\mathrm{Mn}^{3+}$ and $\mathrm{Al}^{3+}$

$\left(r_{\mathrm{Mn3}+}=0,66 \AA, r_{\mathrm{Fe} 3+}=0,64 \AA\right.$ and

$\left.r_{A / 3+}=0,51 \AA\right)$. These values justify the main substitution of $\mathrm{Fe}^{3+}$ by $\mathrm{Mn}^{3+}$, and the minor one of $\mathrm{Al}^{3+}$ by $\mathrm{Mn}^{3+}$.

On the other hand, a little amount of $\mathrm{Mn}^{2+}$ is present occupying $\mathrm{Ca}^{2+}$ positions in both compositions. The differences of $\mathrm{Mn}^{2+}$ contents in two compositions $\left(\mathrm{Ca}_{4} \mathrm{Fe}_{2-x} \mathrm{Mn}_{x} \mathrm{Al}_{2} \mathrm{O}_{10}\right.$ and $\mathrm{Ca}_{4} \mathrm{Fe}_{2} \mathrm{Al}_{2-x} \mathrm{Mn}_{x} \mathrm{O}_{10}$ with 0,030 and $0,078 \mathrm{mols}$ of $\mathrm{Mn}$, respectively) may be due for the different oxidizing conditions of the kiln, because it was used two different kiln of laboratorie.

\section{CONCLUSIONS}

1.- Mn solid solubility on the $C_{3} S$ and $C_{2} S$ structures are 0,72 $\pm 0,11$ and 1,53 $\pm 0,12$ $\mathrm{Mn}$ (wt\%), respectively. The formula proposed for these solubilities are: 


$$
\begin{aligned}
& \mathrm{Ca}_{3}\left(\mathrm{Si}_{0,954} \mathrm{Mn}_{0,036}\right) \mathrm{O}_{5} \\
& \mathrm{Ca}_{2}\left(\mathrm{Si}_{0,946} \mathrm{Mn}_{0,048}\right) \mathrm{O}_{4}
\end{aligned}
$$

2.- La solubilidad sólida del $\mathrm{Mn}$ en la estructura del $\mathrm{C}_{3} \mathrm{~A}$ es de $0,78 \pm 0,12 \%$ en peso. La fórmula propuesta para esta solubilidad es:
2.- Mn solid solubility on $C_{3} A$ structure is $0,78 \pm 0,12 \mathrm{Mn}$ (wt\%). The formulae proposed for this solubility is:

$$
\left(\mathrm{Ca}_{2.984} \mathrm{Mn}_{0,016}\right)\left(\mathrm{Al}_{1,979} \mathrm{Mn}_{0.021}\right) \mathrm{O}_{6}
$$

3.- Las solubilidades sólidas del $\mathrm{Mn}$ en las estructuras del $\mathrm{C}_{2} \mathrm{~F}_{3} \mathrm{C}_{4} \mathrm{AF}$ son $6,80 \pm 0,87 \%$ y $6,73 \pm 2,51 / 6,82 \pm 0,31 \%$ en peso. Las fórmulas propuestas para estas solubilidades son:
3.- $M n$ solid solubility on $C_{2} F$ and $C_{4} A F$ structures are $6,80 \pm 0,87 \mathrm{Mn}(\mathrm{wt} \%)$ and $6,73 \pm 2,51 / 6,82 \pm 0,31 \mathrm{Mn}$ (wt\%). The formula proposed for these solubilities are:

$$
\begin{aligned}
& \left(\mathrm{Ca}_{1,969} \mathrm{Mn}_{0,031}\right)\left(\mathrm{Fe}_{1,696} \mathrm{Mn}_{0,304}\right) \mathrm{O}_{5} \\
& \left(\mathrm{Ca}_{3,970} \mathrm{Mn}_{0,030}\right)\left(\mathrm{Fe}_{1,668} \mathrm{Mn}_{0,332}\right)\left(\mathrm{Al}_{1,767} \mathrm{Mn}_{0,233}\right) \mathrm{O}_{10} \\
& \left(\mathrm{Ca}_{3,922} \mathrm{Mn}_{0,078}\right)\left(\mathrm{Fe}_{1,679} \mathrm{Mn}_{0,321}\right)\left(\mathrm{Al}_{1,797} \mathrm{Mn}_{0,203}\right) \mathrm{O}_{10}
\end{aligned}
$$

\section{BIBLIOGRAFIA}

\section{REFERENCES}

(1) Lea, F. M. and Desh; C. H. The Chemistry of Cement and Concrete. 2nd edition. 76-78, (1956).

(2) Essulescu, M. VI International Congress on the Chemistry of Cement (Moscú) (1974).

(3) Knöfe!, D.; Strunge, J. and Narubuer, H. V. Zem. Kalk Gips 12, 651-655 (1984).

(4) Gutt, W. and Osborne, G. J.: Trans. Brit Ceram. Soc. 68, 129 (1969).

(5) Sakurai, T.: Review of the 14 General Meeting, Japan. Cement Engineering Assoc. Tokyo, 23-24 (1960).

(6) Akatsu, K. Maeda, K.: Review of the 21 General Meeting, Japan Cement Engineering Assoc. Tokyo, 34-37 (1967).

(7) Parker, T. W.: III International Congress of Chemistry of Cement (London) (1952).

(8) Akatsu, K. Monna, I.: Review of the 20 General Meeting. Japan Cement Engineering Assoc. Tokyo, 41 (1966).

(9) Puertas, F.: Tesis Doctoral. Universidad Autónoma de Madrid (1987). 